Fizika Nizkikh Temperatur, 1998, v. 24, No 2, p. 148-157

\title{
Low temperature physics without a cryostat: laser cooling and trapping of atoms
}

\author{
N. P. Bigelow \\ Department of Physics and Astronomy, Laboratory for Laser Energetics and The Materials Science Center \\ The University of Rochester, Rochester, 14627 Now York, USA \\ E-mail: nbig@lle.rochester.edu
}

\begin{abstract}
In recent years, there has been significant interest in the laser manipulation of neutral atoms, and specifically in the use of light fields to manipulate not only the internal but also the external coordinates of an atom. Among the most dramatic results of this work have been the production of dense ultra-cold samples of trapped neutral atoms. These samples have been used to expand the horizons of fundamental physics and have made possible significant advancements in metrology. Furthermore, the ability to manipulate atomic motion with light has helped to create entirely new fields of research such as atom optics where matter wave analogues of light wave systems are investigated and optical where light is used to create a periodic array of atoms similar to crystals.
\end{abstract}

PACS: 03.75.Fi, 32.80.Pj, 42.50.Dv

\section{Laser cooling: a historical view}

The idea of manipulating atomic motion through the basic properties of the atom-field interaction represents a significant change in the paradigm of atomic physics. At the turn of the century, as physicists began to explore the idea which we now describe under the heading «modern physics», the focus of attention in describing the atom-field interaction was on the changes that photon absorption and emission caused in the atom's internal coordinates (the electronic quantum numbers). Energy conservation in the process is key; the change of the electronic energy must equal that of the absorbed or emitted photon $\left(E_{\mathrm{ph}}=h f_{\mathrm{ph}}\right)$ and the selection rules for various possible and forbidden transitions reflect the constraint of the conservation of angular momentum of the atom+laser field system. Starting with de Broglie, the idea that a photon carries linear momentum $(p=h / \lambda)$ as well as angular momentum was well established. However, it was not until the mid-to late 1970 s that the consequence of linear momentum transfer in the atomfield interaction was considered as a viable means of manipulating atomic velocities. By the mid 1980s this possibility had been experimentally demonstrated and by the 1990 dozens of laboratories around the world were carrying out experiments on laser cooled and trapped atoms. Temperatures of microKelvins were being measured, and the very nature of the center-of-mass motion of the atom was shown to need a fully quantum treatment to explain many observed effects. At the time of this Workshop, the physics of laser cooling and atom trapping has already had an impact on a variety of problems: compact cold-atom clocks have been built which compete in accuracy with the worlds best time standards and are cheaper and more compact; novel forms of materials fabrication have been demonstrated and are under vigorous investigation in both academic and industrial settings; ultra-high precision gravitometers and accelerometers have been built and are constantly being improved. And these examples are only a small sample of the variety of work underway in LCAP laboratories.

\section{What is the light pressure force?}

There are two essential ideas which can be used to provide a fairly complete answer to this question. One relates to processes in which an atom absorbs a photon and the reemits it some characteristic time $\tau_{\mathrm{sp}}$ later. The other relates to processes in which stimulated emission must be accounted for. In most situations the force experienced by a two level atom interacting with a monochromatic light field can be expressed as $F=F_{\text {sp }}+F_{\text {st }}$ where the first and second terms reflect the spontaneous and stimulated components of the force, respectively. 
Stimulated forces arise from the interaction between the quadrature component of the dipole moment induced in the atom by the field and the gradient of the field. Unlike $F_{\text {sp }}, F_{\text {st }}$ is a conservative force and is often referred to as the dipole or gradient force because it changes sign with detuning and because it depends on the gradient of the field intensity. Because the dipole force is conservative, it can be expressed in terms of a potential $U(x)=$ $=-h \Delta \ln [1+p(x)]$ where

$$
p(x)=I(x) / I_{\text {sat }}(2)(\Gamma / 2)^{2} /\left[\Delta^{2}+(\Gamma / 2)^{2}\right],
$$

$I_{\text {sat }}$ is the two-level saturation intensity. Hence, for moderate field intensities or large detunings (compared to $\left.I_{\text {sat }}\right) F \sim \nabla I(x)$.

The spontaneous force is the workhorse of many laser cooling experiments and so-called optical molasses. $F_{\mathrm{sp}}$ can be understood in terms of the momentum transferred to an atom as photons are absorbed and reemitted spontaneously. When an atom absorbs a photon it receives a momentum kick and recoils along the wavevector $\mathbf{k}$ of the absorbed photon. If the subsequent reemission is via spontaneous emission, then the emission direction is randomly oriented in space. Averaged over many events, the momentum transfer to the atom on emission averages to zero, whereas the momentum transfer on absorption is cumulative, producing a finite average force along $\mathbf{k}$. The size of this force is of the order of the recoil momentum per absorption $h \mathbf{k}$ multiplied by the transfer rate (the inverse of the excited state lifetime for the driven transition). For sodium, this corresponds to about $10-20 \mathrm{~N}$, or an acceleration of $10^{6} \mathrm{~m} / \mathrm{s}$ ! Note that the length scale over which $F_{\text {sp }}$ varies is determined by the variations in $\mathbf{k}$ (particularly its direction) and by the spatial variations in the field intensity.

If the light field is an optical standing wave, and the field frequency is tuned below (to the red off) the two level transition frequency, then spontaneous processes can give rise to a damping of atomic velocities. The velocity damping (cooling) arises from the fact that an atom with a finite velocity will be Doppler shifted into resonance with the traveling wave component of the standing wave which is directed opposite to the atomic motion. The atom will therefore absorb and reemit more photons from this wave than a slower atom with a smaller Doppler shift. Clearly then, the faster atom will generally experience the larger force. The result is a velocity dependent photon scattering rate. Moreover, this velocity dependent force will be directed opposite to the atom's velocity, causing the atom to decelerate. This configuration is referred to as optical molasses and can readily be generalized to three dimensions. The «terminal» velocity or temperature for an atom in equilibrium with optical molasses is determined by the balance between the Doppler cooling and the spontaneous or diffusive heating associated with the individual random velocity recoil kicks experienced during spontaneous emission. In other words, by equating the time averages: <heating due to diffusive random spontaneous kicks $>=<$ cooling rate in molasses $>$. In the limit where the atom is modeled as a simple twolevel system (ground and excited states) it is expected that the atomic sample will equilibrate at the so-called Doppler temperature $T_{D}=h \Gamma / 2 k_{B}$ which, for sodium, is $240 \mu \mathrm{K}$. To the surprise of many researchers, it was discovered by the group at NIST [1] that, in fact, the atoms in optical molasses were cooled to well below this temperature by the molasses. More recently, for Cs atoms, temperatures below $2 \mu \mathrm{K}$ (barely a few photon recoil kicks of average momentum!) have been achieved using optical molasses. The microscopic explanation for this remarkable supercooling power of optical molasses involves two facts: first, the atoms are rarely two-level systems, and usually a complex magnetic substructure is present and cannot be neglected. Second, there are complex spatially varying intensities and polarizations of a three-dimensional optical molasses which cannot be neglected. These two facts, coupled with the effects of optical pumping give rise to a new time scale in the cooling problem: the internal time scale for motion of population amongst these internal states. The results is a beautiful mechanism [2], now frequently referred to either as polarization gradient or «Sisyphus» cooling which explains the deep cooling. It is important to recognize the temperatures used to describe these laser cooled gasses are true kinetic temperatures. In fact, the gas temperature is often measured by analyzing the ballistic motion of the atoms in the absence of the optical trap using a time-of-flight technique.

\section{Clock-type measurements and fundamental physics}

Traditionally, one of the most important goals of research in atomic physics is the measurement of fundamental constants of nature. Among the most important atomic physics «devices» is the high-precision atomic clock. These clocks not only provide the standard by which we tell time, but are crucial to the way we communicate and navigate on earth and in the air: Without question, atomic clocks affect almost every aspect of modern life. Two vital 
concerns in the conception of such ultra-high precision devices are: (1) that the precision is inversely proportional to the observation time (this stems from the uncertainty relation $\Delta E \Delta t \geq h / 2$ ) and (2) that the first and second order Doppler shifts associated with atomic motion can cause broadening of the atomic transition, thereby compromising the measurement accuracy and precision. Laser cooled atoms have significantly pushed back both of these limitations. This is because these atoms move very slowly and because they remain in a given observation volume for very long times. Indeed, compact cold atom clocks have already been built and have provided a short term stability which is significantly better than the commercial standards. Nevertheless, observation times in these clocks are still set by gravity: ultimately, the atoms fall out of the experiment under their own weight! Increased observation times are possible in microgravity and can result in at least one or two orders of magnitude further improvement. A long-term accuracy near a part in $10^{17}$ is expected - making these clocks three orders of magnitude better than accepted standards and several additional orders of magnitude better than current space qualified clocks!

\section{The atomic fountain clock}

The atomic beam frequency standards currently maintained by NIST and other laboratories around the world are based on measurements made using what is referred to as the Ramsey separated zone technique. Common atoms for use in these clocks are atomic rubidium ( $\mathrm{Rb}$ ) and cesium ( $\mathrm{Cs}$ ). Here the transitions between two hyperfine ground states form the atomic «reference frequency». Consider for example cesium (see Fig. 1). To probe this ground state level splitting, transitions between $g 1$

Fig. 1. The ground state hyperfine splitting of atomic cesium.
Fig. 2. A sketch of a cold-atom atomic fountain clock.

and $g 2$ are induced as the atoms pass through a pair of spatially separated microwave cavities which are aligned along the atomic beam axis. In this type of separated zone measurement, the performance of the clock is strongly determined by the integration time which is set by the time-of-flight of the atom between the two microwave cavities; the longer the time, the more accurate the final measurement.

In the cold-atom fountain clock, a significantly different geometry is used. First, a laser cooled sample of atoms is prepared and positioned below a single microwave cavity. By pushing on the bottom of the ultra-cold atomic sample with a pair of laser beams oriented along the vertical axis (see Fig. 2), the sample is launched upward, against the force of gravity, such that it passes through the cavity. The atoms then slow due to the acceleration of gravity, turn around, and eventually fall back downward through the microwave cavity. In this way, the atoms pass through the cavity twice and an effective «two-zone» measurement is made using only one true interaction zone.

One obvious advantage of the cold-atom fountain clock is that a two-zone measurement can be made with only one cavity. This leads to a reduction in the amount of hardware and has the additional advantage that systematics associated with possible differences between the cavities are reduced. However the most important advantage of the cold-atom 
fountain clock is that substantially longer integration times can be realized and that this can be achieved using a much smaller apparatus. For example, using a hot atomic beam ( $400 \mathrm{~K})$ the typical measurement integration times are $\sim 10 \mathrm{~ms}$ for a $2 \mathrm{~m}$ cavity separation whereas, for the cold source $(<1 \mathrm{~K})$, an integration time of $\sim 0.5 \mathrm{~s}$ is possible with a clock for which $h=0.3 \mathrm{~m}$ ! Using a cold-atom clock based on a laser cooled sodium vapor, the group at Stanford has reported [3] Ramsey fringe width measurements of $\sim 2 \mathrm{~Hz}(\sim 100$ times narrower than typical in «hot» atom clocks). Similar devices have been investigated using cesium atoms [4] yielding a $\delta v / v_{h f}$ of $\sim 10^{10}$. From this type of device, a meaningful performance figure of merit is given be the Allan variance $\sigma \approx 3 \cdot 10^{-14} / \sqrt{\tau}$ or $10^{-16} /$ day [5]. (This is a vast improvement over typical flight qualified clocks, which perform at a $10^{-12}$ level, and commercial atomic frequency standards which perform at about $10^{-14}$ !) In microgravity, one can expect to take the performance of the cold-atom clock even further. In the terrestrial fountain clock, the interaction time is set by the size of the apparatus and most specifically by the fountain height $h$. In particular, $T_{\text {int }} \sim \sqrt{h}$. In microgravity, the interaction times will be set instead by the ballistic expansion of the atoms within a single observation volume. An increase of performance to $\sim 10^{-17}$ / day can be expected if $\mathrm{nK}$ temperature atoms are used. Experimental investigations of microgravity clocks have already begun in France [6] and a vigorous effort on cold-atom clocks is also currently underway within the NASA Microgravity Fundamental Physics program.

In order for cold atom space clocks to reach their full potential, one important problem that will have to be addressed will involve the management of collisional frequency shifts. Such shifts are a particularly important problems for fountains based on ultracold atoms because in the low temperature limit s-wave scattering dominates and collisional crossections begin to scale as $\lambda_{d B}^{2} / \pi$ (where $\lambda_{d B}$ is the thermal de Broglie wavelength which increases with decreasing temperature). Indeed, the Stanford group have already used a cold atom fountain clock to directly measure $\mathrm{mHz}$ level collisional shifts [7]. Such shifts may not present fundamental limitations for cold-atom clocks, however, and several novel schemes utilizing coherent interference effects and/or multi-color laser excitation techniques have already been proposed to circumvent collisional shifts. Indeed, experiments are already underway to implement and test these strategies.

\section{Fundamental clock-type experiments in space}

Almost every measurement made in science ultimately traces to a time or frequency measurement (thermometry, distance measurement, pulsar period timing, etc.) and both terrestrial and deep space navigation rely crucially on precision clocks. However, the introduction of ultra-high precision cold atom clocks into the microgravity program is of deeper significance. At the heart of the atomic clock is a measurement of a carefully chosen energy level splitting within the atom. These splittings are not only nature's own reference «standards» but, at the resolution which can be expected using microgravity clocks, the splittings become measurably sensitive to subtle aspects of fundamental forces and to basic symmetries of nature. Usually, these last classes of questions are the realm of high-energy or «particle» physics. Ultra-high precision atomic measurements, however, can now make important contributions to answering these questions and microgravity fundamental physics can play a vital role in this contribution. For example, parity nonconservation experiments in atomic cesium already compete with accelerator based measurements for the determination of the Weinberg angle, a direct measure of the electro-weak mixing angle in the Weinberg-Salam model (and a number which determines the ratio of the $W$ to $Z^{\circ}$ boson masses). As with any clock type measurement, one important limitation of these experiments remains the observation time. It is natural then that these experiments should benefit from the use of laser cooled atoms and should benefit even more so from the microgravity environment of space.

\section{Electric-dipole moment}

A experiment closely related to PNC is the search for the permanent electric-dipole moment (EDM) of the electron. The existence of an EDM can be traced directly to $\mathrm{T}$ and/or $\mathrm{CP}$ violating interactions, or, in other words, to the most basic symmetry properties of nature. The EDM measurement consists of a very precise determination of the response of the atomic level splitting to an applied electric field. Essentially, this is a clock-type measurement: a state is prepared and allowed to evolve in the presence of an applied electric field. After some period the populations of the atomic states are then probed and used to determine level shifts and spacings. Using microgravity, a cold-atom EDM apparatus is predicted to provide at least two orders of magnitude of improvement over the current EDM measurements (to date these measurements have placed an upper bound on the possible mo- 
ment). Regardless of the exact outcome of an improved EDM experiment, the results are essentially guaranteed to be significant: If this experiment detects no moment (with better than a precision of $\sim 10^{-17} \mathrm{e}-\mathrm{cm}-$ the expected accuracy of a microgravity EDM experiment) then several theories competing with the Standard Model can be eliminated: supersymmetry, flavor changing, etc. On the other hand, if a moment is detected, far reaching revisions in the foundations of physics will be required. The significance of such fundamental LCAP experiments is currently reflected in the support of ground based EDM experiments within the current Microgravity Fundamental Physics program.

\section{General relativity}

The clock itself reaches deeply into the foundations of physics. Clocks are central in the realm of general relativity and in questions concerning the very nature of gravity itself. Here the motivation for space based clocks is not only tied to the improved performance expected in a microgravity environment but because these clocks will have access to fundamentally different positions in space-time than are available on earth. An important example of this latter physics is that revealed in the comparison of an earth based clock with a space based clock. This comparison provides a direct measurement of the gravitational red-shift. Indeed, improvements of one to two orders of magnitude of the current accuracy of 100 parts per million can be expected using space based ultra-high precision clocks. Measurement of the gravitational red-shift test important aspects of the local position invariance part of Einstein's Equivalence principle and is tied to the possible spatial variation of the finestructure constant $\alpha-$ a parameter central to quantum electrodynamics.

\section{Multiple clock experiments}

The motivation for developing cold-atom clocks for use in a space environment goes beyond a desire for absolute performance. If performance were the only goal, then the demand would be to develop the best possible space qualifyable clock with no regard for laser cooling and the clocks underlying physical mechanism. However, there are important reasons for which this is not the case. The first example are the clock-type measurements such as the search for the permanent dipole moment of the electron, as discussed above. There is no direct analogue of these experiments that can be carried out using ion clocks or superconducting cavity oscillators. However, there is a second important point: there is an important class of experiments that can be carried out only if there are a multiplicity different types of high precision clocks available in space. This class of measurements is based on systematic measurement the mutual drift of clocks based on vastly different physical mechanisms. Indeed, such a measurement was identified as an important issue earlier this century by leading physicists such as P.A.M.Dirac. The basic idea is that different clock designs can be sensitive to different fundamental forces. Consider, for example, the atomic clock. Here the hyperfine splitting (the frequency reference) is determined by both nuclear interactions (through the contact hyperfine interaction) and by electromagnetic interactions. By contrast, in many designs of flywheel type clocks, such as the high- $Q$ microwave resonator, the frequency is set by some mechanical dimensions of the clock and hence is mostly determined by electromagnetic forces and gravitational stresses. As a results, a comparison of the relative drifts of these clocks in a microgravity experiment could give unique insight into the relative drifts of the strong and electromagnetic forces.

\section{Atom optics and atomic interferometry}

Atom optics is the field of research aimed at the development of devices and techniques for manipulating atoms in direct analogy with the systems used to manipulate light. Although progress in atom optics has not been limited to work with laser cooled atoms, the motivation for using cold atoms is clear: as the temperature is decreased, the de Broglie wavelength of the atom increase and so the wave nature of the atom emerges more and more dramatically in terms of the atomic center of mass motion. One of the hallmarks of progress in atom optics has been the development of the atom interferometer.

Conceptually, the atom interferometer is essentially identical to the light interferometer. An input beam is caused to split into two or more components which propagate over some distance in space and are then caused to recombine. If the net path traveled by one component differs from that traveled by the other, there will be a phase shift associated with the different path lengths and interference effects between the components can be observed when they recombine. In the atom interferometer, the splitting and subsequent recombination of the atomic wavepacket has been accomplished using a variety of techniques. Both nanofabricated micro-structures (which act as amplitude gratings) and standing wave laser fields (phasegratings) have been used as beam splitters. In 
Fig. 3. The «three-level» atom (left). A depiction of the atomic momentum before and after the application of the first laser pulse (right). The two components of the wavepacket have different vertical momenta and therefore separate in space. Under the influence of gravity, these atoms slow as the move upward and the laser frequency must be adjusted to account for this velocity change. A measurement of the velocity change can be used to determine the acceleration due to gravity at the location of the experiment.

addition, an interferometer using time dependent light (pulsed) fields has been realized. The interest in atom interferometers is both fundamental - in terms of the investigation of interference of massive composite particles which possess a rich spectrum of internal degrees of freedom - and is motivated by potential applications. One of the applications most relevant to this workshop is the absolute measurement of the acceleration due to gravity [8]. In these experiments a sample of «three-level» atoms (see Fig. 3), is initially cooled and confined in an optical trap. The sample is then prepared in one of the two ground states and next it is irradiated with a pair of couterpropagating optical pulses (see Fig. 3, right). The light pulses are configured to act as $\pi / 2$ pulse, transferring half of the population to the other ground state. The resulting state of the atom is a wavepacket which is a superposition of two internal states ( $g 1$ and $g 2$ ) that differ by two photon recoil's worth of momentum. These two components of the atomic wavefunction therefore spread apart in space. In this way, the pulses serve as the beam splitter for the atom. Some time later a second pulse is applied in order to reverse this process and to cause the atomic wavepackets to interfere. Because the atoms simultaneously move under the acceleration of gravity, it becomes neces- sary to adjust the laser frequencies in order to keep in resonance with the atoms - if the frequency is adjusted to keep the atoms in resonance with the lasers, there will be no net phase shift when the atoms recombined. By measuring the frequency shift required, the acceleration of gravity can be measured. In the Kasevich experiment, the absolute value of $g$ was measured to a part in $10^{-6}$ ! In a related experiment, the single photon recoil momentum was measured to a part in $10^{-7}$ ! .

\section{Colder denser samples: other cooling and trapping schemes}

\section{Getting colder}

The most common scheme for laser cooling and trapping atoms is the magneto-optical trap [9]. In this trap, a static magnetic field is combined with a three-dimensional optical molasses to yield both cooling (a damping of atomic velocities) and trapping (a restoring force about some fixed point in space). However, in the quest to achieve ever lower temperatures, cooling schemes which involve optical molasses (and hence which involve spontaneous emission) have one basic limitation: the lowest energy scale for the atom will be set by the single photon recoil energy. It is important to recognize 
that the maximal advantage of a microgravity environment for many LCAP experiments will be realized with the coldest possible atoms. For this reason, the cooling of atomic vapors below the recoil limit is of interests to this workshop.

To break this fundamental barrier - the single photon recoil limit - several techniques have already been investigated. One very elegant technique is referred to as velocity selective coherent population trapping [10]. This technique involves using laser fields to create a coherent superposition state of the atom which effectively turns-off the atom's ability to fluoresce (these states are named «dark-states»). Using this approach, kinetic temperatures below the single photon recoil have already been achieved [11]. However, the technique has been less successful at increasing the phase space density of the atomic samples (density in configuration space and momentum space).

\section{Conservative traps and evaporative cooling}

A second strategy involves the deep cooling of the atoms by evaporation. In this scheme, the hottest atoms in the distribution are allowed to preferentially escape from the sample, leaving only the cold atoms behind. The basic ingredients for evaporative cooling are a conservative trap potential in which the atoms can be suspended and a means for removing or «evaporating» the most energetic atoms in the sample. Two popular schemes for creating such a potential are the magnetic trap and the dipole trap.

In the magnetic trap (see Fig. 4) a force is exerted on the atoms by coupling to the atomic magnetic moment. Depending on the alignment of the magnetic moment with respect to the field, there is an associated potential energy $U=-\mu \cdot \mathbf{B}$. If a field configuration is used in which $\mathbf{B}$ varies in space, then there will be a gradient in this potential and hence a force on the atom. To induce evaporation an rf field $\omega$ is applied to the sample which

Fig. 4. The magnetic trap and rf forced evaporative cooling.
Fig. 5. Dipole (FORT) trap made from focused laser beam tuned below the atomic resonance frequency.

induces atomic spin-flips. When the spin (magnetic moment $\mu$ ) of the atom is flipped, the magnetic energy for that atom changes sign, and the magnetic force on the atom ejects that atom from the trap. The technique can be used to select out the hottest atoms in the sample because the rf resonant frequency needed to flip the atomic spin is determined by the magnitude of the field $\mathbf{B}$ at the atomic coordinate, which is in turn determined by the position in the potential and hence by the kinetic energy (the temperature) of the atom. This technique for deep cooling has recently been used to increase the phase space density of cooled and trapped atoms to the point where Bose-Einstein condensation has recently been observed in a trapped atomic vapor.

\section{Dipole traps and the FORT}

An alternate trapping scheme is the far off resonance dipole trap (FORT) [12]. In this scheme (Fig. 5) the electric dipole force $(F=-\nabla(\mathbf{p} \cdot \mathbf{E})=$ $=-\nabla U_{\text {dip }}(x)-$ see above $)$ is used to confine the atoms in a tightly focused laser beam. By varying the laser beam parameters, the depth of the confining potential can be modified and evaporative cooling can be made to occur [13]. The dipole and FORT traps has also been used to carry out many elegant experiments on ultra-cold collisions among the trapped atoms [14].

\section{Gravity?}

In addition to providing a potential from which the atoms can be evaporated, a prime raison d'etre for each of these traps is to balance the effects of gravity: from the point of view of precision spectroscopy, the presence of the trap force can significantly perturb the sample, giving rise to incipient frequency shifts, distortions of the atomic cloud. 
Furthermore, the presence of gravity introduces limitations an the accuracy of ballistic expansion measurements and will limit the ultimate efficacy of additional deep cooling techniques (not discussed here) such as adiabatic expansion cooling.

\section{Bose-Einstein condensation: new phase transitions, new states of matter and new superfluids}

Perhaps one of the most dramatic developments of the 1990s has been the realization of Bose-Einstein condensation (BEC) in a dilute atomic vapor. Bose condensation is a phase transition that occurs in a gas of identical bosons when the interparticle spacing becomes comparable with the thermal de Broglie wavelength. At this point, the particles are no longer quantum mechanically distinguishable and a correct quantum treatment of the gas is made in terms of a fully symmetrized macroscopic wavefunction. From a statistical point of view, the condensate represents the macroscopic occupation of a single quantum state, usually thought of in terms of the ground momentum state of the system. The physics of these Bose condensed vapors is intimately tied to the physics of quantum fluids studied in the field of low temperature physics. Soon, BEC experiments will require microgravity and a strong synergy with related work in low temperature physics should come naturally. However, the need for microgravity BEC experiments may also be driven independently by efforts to achieve lower sample temperatures and higher densities. In particular, as noted earlier, forced evaporative cooling depends on the spatial distribution of the hot tail of the trapped atom sample and the shape of this distribution near the classical turning point in the confining trap potential. As the sample becomes colder and colder, and as the sample moves closer and closer to the bottom of the trap well, the atomic distribution will be distorted by gravity an effect which will disrupt the ability to control the cooling process.

In the current experiments, the pathway to BEC begins with a laser cooled sample $\left(\sim 10^{10}\right.$ atoms at $\sim 20 \mu \mathrm{K})$. This sample is then loaded into a magnetic trap and evaporatively cooled until the system condenses. In the earliest experiments [15] this occurred at about $170 \mathrm{nK}$ for $10^{3}$ atoms. In recent experiments sample sizes in excess of $10^{6}$ atoms have been condensed. The condensate in this work has been detected by releasing the atoms and examining the free expansion of the condensed cloud in order to determine the momentum distribution of the sample.
One of earliest successes of trapped alkali BEC has been in the excellent agreement between the experimental data and the theoretical results derived using a mean-field theory. A representation of the condensate wavefunction in the mean field picture can be derived from the Gross-Pitaevskii (GP) equation. Essentially, this is a Schrödinger equation for the many-particle ground state (the BEC) with an additional term in the Hamiltonian which is of the form: $U_{0}|\Psi|^{2}$. Here $\Psi$ is the BEC wavefunction and $U_{0}$ is the interaction potential between the atoms in the condensate. In many situations $U_{0} \sim a_{s}$ where $a_{s}$ is the $s$-wave scattering length of the particles. Frequently it is simplest to solve the GP equation in the so-called Thomas-Fermi approximation which essentially means that the kinetic energy term of the Hamiltonian can be neglected. At Rochester, we have developed a new numerical technique based on a variational approach for solving the GP equation without making any approximations [16]. In most situations, the modifications to the shape of $\Psi$ due to the inclusion of the kinetic energy are small. In fact, only in regions where there is a large curvature in $\Psi$ (and hence a large contribution from the $\nabla^{2} \Psi$ term in the Hamiltonian) does the Thomas-Fermi approximation cause problems. It is not surprising that these problems can become most marked near the condensate boundaries.

With the advent of multi-species traps and the recent realization of a Bose condensate comprised of two different spin-states of a vapor of rubidium atoms [17] a new and important question has arisen: what is the nature of a two-species Bose-Einstein condensate - the 2BEC? In approaching this question it is important to realize that we cannot expect that each condensate will simply co-exist with the other condensate. The reason for this is that, even though the alkali BECs are very dilute $\left(\sim 10^{13}\right.$ atom/cc) the role of interparticle interactions is still crucial. For a $2 \mathrm{BEC}$, then, we must consider three classes of interactions: the two selfinteractions of each species and a third «cross-species» interaction. In the mean field limit, this means that the 2BEC can be characterized by two coupled GP equations and three scattering lengths $a_{11}, a_{22}$, and $a_{12}$ (the cross species scattering length) [17].

A rich array of new phenomena have been predicted for this system including modifications to the ground state wavefunction profile, the excitation spectrum and even the essential stability of the condensate itself. We have been investigating the 2BEC using a modification of our variational technique [16]. In Fig. 6 we show the ground state 
Fig. 6. The ground state density profiles of a two-species BoseEinstein condensate (2BEC) for two different choices of crossscattering length $a_{12}$. For this figure we consider a mixture of sodium (Na with $\left.a_{11}=3 \mathrm{~nm}\right)$ and rubidium $\left(a_{22}=6 \mathrm{~nm}\right)$ atoms.

condensate wavefunction for a mixture of sodium atoms and rubidium atoms contained in a spherically parabolic trap. The parameter which is varied between the different parts of this figure is the cross scattering length $a_{12}$. We see that for strong repulsive interactions (i.e., $a_{12}>0$ ) the ground state is definitely not a mixture of two overlapping condensates, but that instead the system has phase separated into two distinct condensates. Because the condensate wavefunction in the mean field is the state which minimizes the mean field energy we can provide a physical interpretation of the phase separation. When the system separates, the overlap between the condensates is reduced, and hence the cross-species mean field energy is decreased. Simultaneously, the outer species must be spread over a larger volume, which decreases its mean field energy. By contrast, the mean field energy of the core increases because the core atoms are now closer together (the core atoms are not «diluted» by the second species). Overall, the total system energy is determined by the interplay of these individual energies and in the phase separated state the total mean field energy is minimized. A careful analysis of the JILA two-spin state condensates has provided some evidence for the effective «condensate repulsion», however a true two-species condensate has yet to be realized. At Rochester, an experimental effort on this problem is well underway.

\section{Perspective}

In many ways, there is a powerful case that the Laser Cooling and Atomic Physics (LCAP) community can benefit greatly from access to space and to a microgravity environment. Fundamental experiments can be and are being envisioned, experiments that can appreciate significant benefit from implementation in space. Furthermore, there are important fundamental LCAP experiments which cannot be realized in a terrestrial laboratory. In a broader framework, it is also clear that LCAP has natural ties to a broad variety of different areas of fundamental science and to the existing programs supported by NASA and other space agencies around the world. In return for the opportunity to carry out research in this new and exciting environment, LCAP research has much to offer to the countries' effort to explore and develop space, contributions ranging from advanced navigation to high accuracy gravitometry. Furthermore, the support of LCAP activities within the NASA microgravity program has the promise to improve life on earth, for example, through better and more compact space based clocks for terrestrial navigation and geology.

1. P. Lett, R. N. Watts, C. I. Westbrook, W. D. Phillips, P. L. Gould, and H. J. Metcalf, Phys. Rev. Lett. 61, 169 (1988).

2. J. Dalibard and C. Cohen-Tannoudji, J. Opt. Soc. Am. B6, 2023, (1989); P. J. Ungar, D. S. Weiss, E. Riis, and S. Chu, J. Opt. Soc. Am. B6, 2058 (1989).

3. M. Kasevich, E. Riis, S. Chu, and R. De Voe, Phys. Rev. Lett. 63, 612 (1989).

4. A. Clairon, C. Salamon, S. Guellati, and W. D. Phillips, Europhys. Lett. 16, 165 (1991).

5. S. Lea, A. Clairon, C. Salomon, P. Laurent, B. Lounis, J. Reichel, and A. Nadir, Phys. Scripta T51, 78 (1994).

6. B. Lounis, J. Reichel, and C. Salomon, C. R. Acad. Sci. Paris 316 Serie II, 739 (1993).

7. K. Gibble and S. Chu, Phys. Rev. Lett. 70, 1771 (1993).

8. M. Kasevich and S. Chu, Phys. Rev. Lett. 67, 181 (1991).

9. E. Raab, M. Prentiss, A. Cable, S. Chu, and D. Pritchard, Phys. Rev. Lett. 59, 2631 (1987).

10. A. Aspect, E. Arimondo, R. Kaiser, N. Vansteenkiste, and C. Cohen-Tannoudji, Phys. Rev. Lett. 61, 826 (1988).

11. J. Lawall, S. Kulin, B. Subamea, N. Bigelow, M. Leduc, and C. Cohen-Tannoudji, Phys. Rev. Lett. 75, 4194 (1995).

12. J. D. Miller, R. A. Cline, and D. J. Heinzen, Phys. Rev. A47, R4567 (1993).

13. C. S. Adams, H. J. Lee, N. Davidson, M. Kasevich, and S. Chu, Phys. Rev. Lett. 74, 3577 (1995).

14. P. Gould, P. D. Lett, P. S. Julienne, W. D. Phillips, H. R. Thorsheim, and J. Weiner, Phys. Rev. Lett. 60, 788 (1988); J. D. Miller, R. A. Cline, and D. J. Heinzen, Phys. Rev. Lett. 71, 2204 (1993).

15. M. Anderson, et al., Science 269, 198 (1995); C. C Bradley et al., Phys. Rev. Lett. 75, 1687 (1995); K. B. Davis et al., Phys. Rev. Lett. 75, 3969 (1995).

16. H. Pu and N. P. Bigelow, submitted to Phys. Rev. Lett. (1997).

17. C. J. Myatt et al., Phys. Rev. Lett. 78, 586 (1977).

18. Tin-Lun Ho and V. B. Shenoy, Phys. Rev. Lett. 77, 3276 (1996); B. D. Esry et al., Phys. Rev. Lett. 78, 3594 (1997); E. V. Goldstein and P. Meystre, Phys. Rev. A55, 2935 (1997). 
The references presented here are by no means exhaustive and neither are they uniform in their distribution over the many important contributions made by groups all over the world. I apologies in advance for omissions of any sort. There are many excellent references, one being the Laser Light Pressure on Atoms, by $V$. G. Minogin and V. S. Letokhov, Gordon and Breach (1986) and another being Laser Manipulation of Atoms and
Ions, CXVIII Int'1. School, Enrico Fermi, E. Arimondo, W. D. Phillips, and F. Strumia (eds.), North-Holland (1992). Because of the rapid advancement in this field, these works is already somewhat outdate and a sample of additional newer results can be found in the bibliographies of the papers listed above. 